\title{
Temperature and time-resolved XANES studies of novel valence tautomeric cobalt complex
}

\author{
S.O. Shapovalova ${ }^{1}$, A.A. Guda ${ }^{1}$, M.P. Bubnov ${ }^{2}$, V.K. Cherkasov ${ }^{2}$, Yu.V. Rusalev¹, V.V. Shapovalov ${ }^{1}$, A.V. Soldatov ${ }^{1}$ \\ ${ }^{1}$ The Smart Materials Research Institute, Sladkova 178/24, 344090 Rostov-on-Don, Russia, \\ ${ }^{2}$ G. A. Razuvaev Institute of Organometallic Chemistry, Russian Academy of Sciences, 49 Tropinina Str., GSP-445, 603950 Nizhny \\ Novgorod, Russia

\section{cherkasova.svetlana.o@gmail.com}

Valence tautomers are characterized by different electron density distributions, where metal-to-ligand electron transfer accomplishes interconversion between tautomers [1]. These compounds are unique model systems that can help study electron transfer mechanisms and find applications as sensors and displays or storage and fast optical switching devices. Wherein the valence tautomeric interconversion can be thermally, magnetically or radiatively driven. Among transition metal complexes cobalt complexes with redoxactive ligands have been shown to undergo a valence tautomeric interconversion between high-spin and low-spin forms [2, 3].

This study is devoted to optical and x-ray structure characterization of novel (N-cyclohexyl-2-iminopyridine)(3,6-di-tert-butyl-obenzosemiquinonato)(3,6-di-tert-butyl-catecholato) (Co2) cobalt complex. We have monitored the transition induced both by temperature and laser stimuli. Complexes were dissolved in toluene. X-ray pump-probe study was performed at the Super-XAS beamline of the Swiss Light Source, Villigen, PSI. A green nanosecond laser with $532 \mathrm{~nm}$ wavelength was operated at $150 \mathrm{kHz}$ repetition mode. We accumulated an X-ray fluorescence signal for different delays after laser excitation pulse with 20 ns time resolution for each energy point. Then principal component analysis was applied for the whole data set.
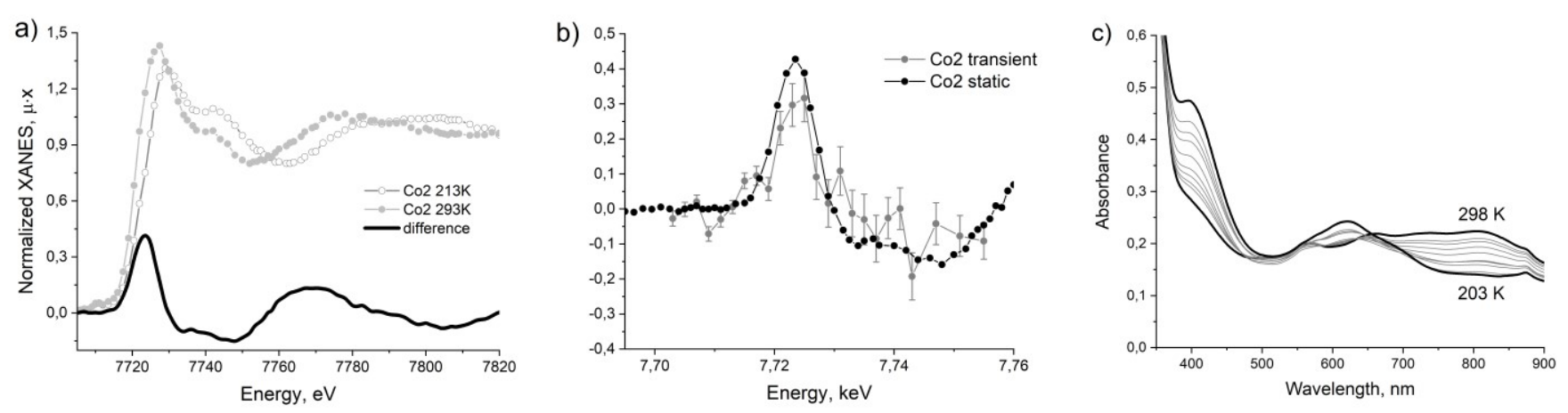

Figure 1. (a) Co K-edge XANES measured for Co2 sample at $213 \mathrm{~K}$ and $293 \mathrm{~K}$ in a solution, (b) transient difference signal after laser excitation for $\mathrm{Co} 2$ and (c) temperature dependence of UV-Vis spectrum for the $\mathrm{Co} 2$ sample in toluene.

Fig. 1 shows XANES and optical results for one of the studied cobalt complexes. According to XANES, change in the cobalt oxidation and spin state can be observed when temperature decreases below $240 \mathrm{~K}$ (Fig.1(a)). The time-resolved transient difference shown in figure $1 \mathrm{~b}$ can be compared to the static difference obtained at low and high temperatures (Fig.1b). Kinetics of the transient signal decay for $213 \mathrm{~K}$ can be approximated by a monotonic exponential decay with a characteristic time of $250 \pm 50 \mathrm{~ns}$. Lowtemperature UV-Vis spectra show intensity decreasing of a broad band at $800 \mathrm{~nm}$ and increase bands at $600 \mathrm{~nm}$ and $395 \mathrm{~nm}$ (Fig.1(c)). The band at $700-850 \mathrm{~nm}$ shows the high spin $\mathrm{Co}^{\mathrm{II}}$ tautomer transition and has likely a metal-to-ligand charge transfer nature. The peak at $\sim 600 \mathrm{~nm}$ characterised the low spin $\mathrm{Co}^{\mathrm{III}}$ tautomer and caused by the ligand-to-metal charge transfer. The appearance of isosbestic points during cooling is strong evidence that only two different species are present in the solution [2]. Obtained results confirm the presence of a valence tautomeric transition in the cobalt complex under study. Our measurements indicate that $\mathrm{Co}^{\mathrm{II}}$ is transformed into $\mathrm{Co}^{\mathrm{III}}$ under temperature decrease while reverse transition can be induced both under the influence of temperatures and laser radiation.

[1] Pierpont, C. G. (2001). Coord. Chem. Rev. 216, 99.

[2] Adams D.M., Hendrickson D.N. (1996). J. Am. Chem. Soc. 118, 11515.

[3] Ash R., Zhang K., Vura-Weis J. (2019) J. Chem. Phys. 151, 104201.

Keywords: Co complex; valence tautomerism; XANES; pump-probe; low-temperature UV-Vis

This work was supported by Russian Foundation for Basic Research, project 20-32-90046. 